\section{Infektiöses Sperma trotz HIV-Therapie}

Auch unter antiretroviraler Therapie ist bei 30\% der HIV-Infizierten die Samenflüssigkeit nicht virusfrei. Dies ist das Ergebnis einer US-Studie mit 101 sexuell aktiven HIV-positiven Homosexuellen. Der Erreger ließ sich außerdem bei $18 \%$ der Männer auch im Blut nachweisen. Diese Werte liegen deutlich höher als in anderen Studien mit nur 2-3\%. Die Ärzte vermuten, dass Zellen des Harnleiters als HIV-Reservoir dienen.

Politich JA et al, AIDS 2012 (online first)

\section{Demenz im Alter rückläufig}

Neue Daten legen nahe, dass sich weitaus mehr Menschen über einen Lebensabend in geistiger Gesundheit freuen dürfen, als zunächst angenommen. Die Hinweise stammen aus einer niederländischen Kohortenstudie, in der die Demenzinzidenz in jeweils zwei Gruppen von über 55-Jährigen im 10-Jahresabstand verglichen wurde. Diese war im Jahre 2000 mit 4,9 pro 1000 Teilnehmer um etwa ein Viertel niedriger als 1990 (6,6 pro 1000). Schrijvers EMC et al, Neurology 2012, 78:1456

\section{Mehr Selbstkontrolle an der Brust}

Säuglinge, die Milch von der Flasche bekommen, legen eindeutig mehr Gewicht zu, als solche, die an der Mutterbrust ernährt werden, so eine US-Studie mit 1899 Babys. Und zwar unabhängig davon ob die Flasche Muttermilch oder Ersatzmilch enthält. Die festgestellte überschüssige Gewichtszunahme pro Monat betrug bei den „Flaschentrinkern" $71 \mathrm{~g}$ bei Formula- und $89 \mathrm{~g}$ bei Muttermilch. Die Ärzte vermuten, dass Säuglinge an der Mutterbrust eine bessere Selbstkontrolle darüber entwickeln, wie viel Milch sie trinken. Li R et al, Arch Pediatr Adolesc Med 2012, 166:431

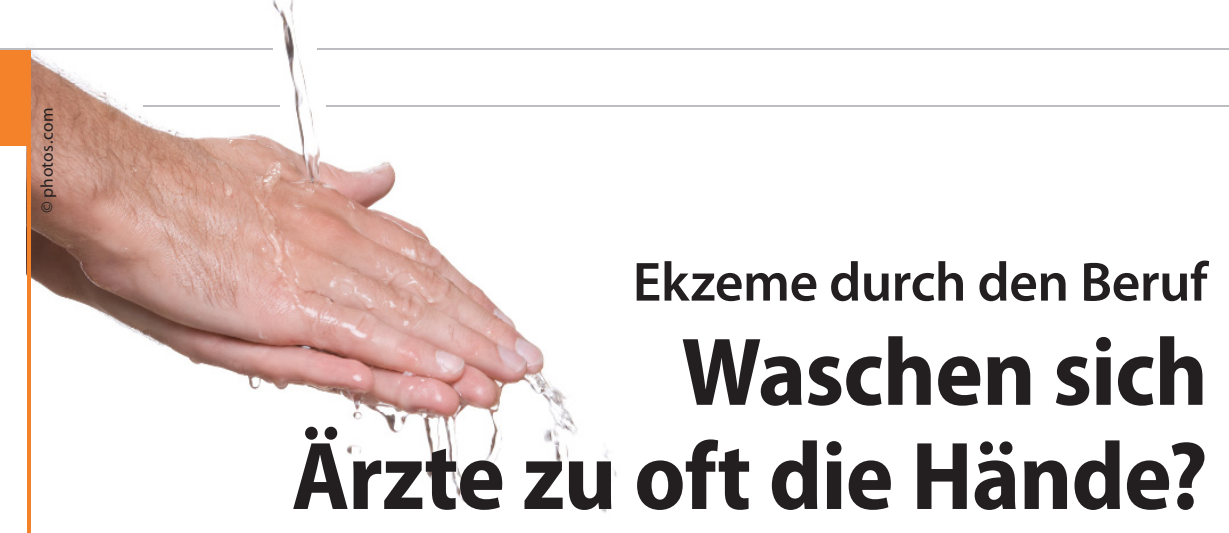

Angehörige von Gesundheitsberufen waschen sich zu häufig die Hände. Auch in Fällen, wo eine einfache Desinfektion genügen würde. Folge sind drei mal so häufige Handekzeme.

Dänische Dermatologen haben 3181 Angehörigen von Gesundheitsberufen dreier dänischer Universitätskliniken einen Fragebogen zu berufsbedingten Hauterkrankungen zugesandt. Daraus ließ sich schließen, dass knapp jeder sechste, an einem Handekzem litt. Als

nächsten Schritt ermittelten die Forscher, welche Faktoren diese Hautkrankheit förderten. Die Teilnehmer, die ein Handekzem hatten, wuschen sich ihre Hände signifikant häufiger als Teilnehmer ohne Ekzem (52\% 10 mal/Tag; 19\% $20 \mathrm{mal} / \mathrm{Tag}$ ).

Allgemein sind Handekzeme bei Medizinberufen mit einer Prävalenz von bis zu $30 \%$ etwa dreimal häufiger als in der Allgemeinpopulation.

$(r b)$

Ibler KS et al, Contact Dermatitis 2012, 66:247

\title{
CME
}

\section{Mit FIN punkten Leser kostenfrei!}

\section{Registrierung und Teilnahmemöglichkeiten}

Falls Sie noch keinen Springer-Medizin-Zugang haben, bitten wir Sie, sich einmalig auf www.springermedizin.de zu registrieren.

e.CME: Kostenfreie Teilnahme bis zum 04. Juli 2012

Gehen Sie auf springermedizin.de/kurse-cme und schalten Sie durch die Eingabe der achtstelligen Fortbildungsidentifikationsnummer (FIN) den Beitrag frei.

Mit Eingabe der FIN gelangen Sie direkt zum gewünschten e.CME-Beitrag.

- e.Tutorial: Teilnahme im Rahmen des e.Med-Abonnements Weitere Infos auf springermedizin.de/eMed

\section{Bitte beachten Sie:}

- Teilnahme nur online unter springermedizin.de/kurse-cme

- Die Frage-Antwort-Kombinationen werden online individuell zusammengestellt

- Es ist immer nur eine Antwort möglich

- Nach erfolgreicher Beantwortung von mindestens 7 der 10 Fragen erlangen Sie 3 CME-Punkte. Diese werden direkt an Ihre Landesärztekammer übertragen, bei Bedarf können Sie sich auch eine Bescheinigung ausdrucken

\section{Kontakt und weitere Informationen:}

Springer-Verlag GmbH

Tel.: 08007780777
Springer Medizin Kundenservice

E-Mail: kundenservice@springermedizin.de 